\title{
TRACKING HUMANS
}

Ben Kröse, Taylan Cemgil, Wojciech Zajdel, Zoran Zivkovic

Informatics Institute, University of Amsterdam, Netherlands

In a human-inhabited environment it is essential that a robot which interacts with humans is able to keep track of them when they move around in the environment. This is not an easy job. Multiple people may be in the robot's vicinity, sometimes a person leaves the vicinity of the robot and reenters some time later. Two issues are essential in tracking: localization and identification. Multi-modal cues (audio, visual, maybe radar) may be used for the task. In this paper we restrict ourselves to visual cues and present our work to track people with a vision system.

The first step in the tracking process is the detection of a human in its field of view. Current research assumes either a static camera, so that everything which moves is categorized as human, or assumes models which make it possible to detect humans with a moving camera. Our approach assumes a static camera for the detection, and uses a background modeling technique. After candidates have been detected, they are tracked with a color-histogram-based object tracking algorithm. A new robust algorithm will be presented that presents a natural extension of the well known 'meanshift' procedure. The new algorithm simultaneously estimates the position of the local mode and the covariance matrix that describes the approximate shape of the local mode. Local features, such as color histograms or spatial features are used for identification.

A second problem occurs if a person disappears and reappears in a camera image. The robot has to answer the question: "is this the person which I saw before or a new person?". To answer this question is not easy, especially if the (light) conditions vary among different observation locations, or if the time between observations is very long (weeks..). The 
color histograms or spatial features used for identification within an image might not be valid anymore. Cognitive reasoning techniques are needed which take into account the context, such as position of the robot, or time between observations. We present a probabilistic framework to deal with this reasoning problem. We show that novel techniques in the field of dynamic Bayesian networks can be used to do fast and reliable tracking in these situations. 